\title{
In memory of Sergey Kullanda (1954-2020)
}

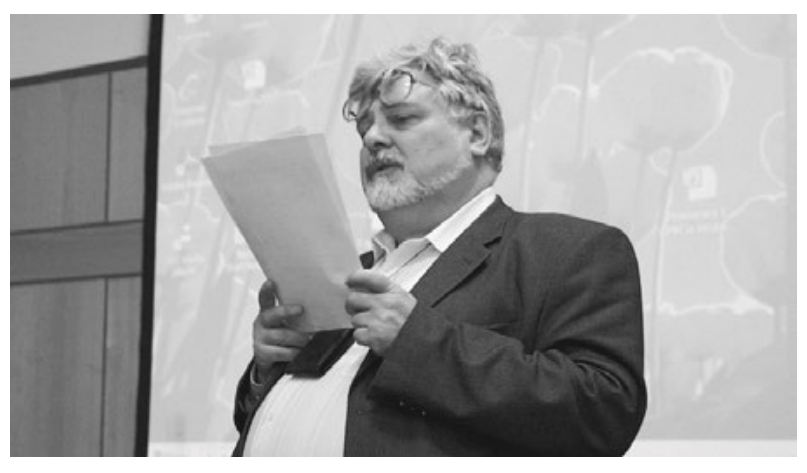

It is with the deepest regret that we announce the loss of our friend and colleague Sergey Vsevolodovich Kullanda, one of the long-term members of the Editorial Board of the Journal of Language Relationship; he passed away on November 30, 2020, after a long and difficult illness.

Sergey Kullanda was first and foremost a historian, specializing in such areas as the history of Indonesia and the ancient Scythian culture. But he had always shared a deep interest in all things related to the history of languages, meaning that most of his research was tied to linguistic issues one way or another.

His studies of ancient social history, driven by a desire to "get to the foundation of each social phenomenon" (p.c.) in the pre-written period, quite logically caused Sergey to fully embrace comparative-historical linguistics. Linguistic data were already quite prominent in his early works on the history of Java and Austronesian studies in general; later on, his focus largely shifted to Indo-European studies and comparative Iranistics, where he used the methodology of linguistic reconstruction to combine anthropological data with the results of studies in diachronic semantics. This was most prominent in his works on the Indo-European system of kinship terms, the main point of those being that the primary semantics of the studied terms must have originally referred not so much to actual kinship as to their social functions, distributed across various age and sex groups. Future research will no doubt show just how close this point of view was to the actual truth; but in any case, Kullanda's interdisciplinary hypotheses were quite immaculately constructed both from the point of view of social anthropology and comparative Indo-European linguistics, and will be highly relevant for subsequent generations of scholars in those fields.

Another of Sergey's favorite topics concerned the role of comparative-historical linguistics in elucidating the ancient history of Iranian and Indo-Iranian peoples. Here must be mentioned his paper on Proto-Indo-Iranian and Proto-Iranian homelands, with a clear and meticulous focus on any potential linguistic contacts between speakers of these protolanguages and other linguistic units; and, most importantly, his 2016 monograph "The Scythians: language and ethnogenesis" (in Russian), in which he has summarized most of the preceding research on Scythian culture, provided a critical evaluation of all pieces of evidence on the Scythian language, established a firm position for Scythian on the genealogical tree of Iranian languages, and scrutinized all possible linguistic contacts between Scythian and the other ancient languages of West Asia and Eastern Europe.

All of these works testify to Sergey Kullanda's huge potential - much of it, alas, unrealised - as a prominent researcher in comparative-historical linguistics, making his passing a truly significant loss for the scientific community at large. This is not even to mention, of course, the significance of this loss for all of Sergey's friends, colleagues, and disciples - many of the latter still remember with fondness his lectures on Sanskrit at the Russian State University for the Humanities. Sergey was one of those true friends one could always count on, and, among other instances, he proved this while serving on the Editorial Board of JLR, never downplaying the importance of even the most routine technical work on submitted papers and sacrificing his personal needs so that the next number of the Journal could be published on time. He will be sorely missed, but never forgotten.

Anna Dybo

on behalf of the Editorial Board of The Journal of Language Relationship

For all those interested in works by Sergey Kullanda, a complete list (in Russian) may be found on the website of the Institute of Oriental Studies of the Russian Academy of Sciences: https:/www.ivran.ru/persons/SergeyKullanda. 